\title{
The Theoretical Model of Social Media's Effect on Brand Preference: From the Perspective of Customer Perceived Value
}

\author{
Yong Ding ${ }^{1, a}$, Jinchuan Xiao ${ }^{1, b, *}$ \\ ${ }^{1}$ School of Management, Hefei University of Technology, Hefei, Anhui, China \\ adingyong@hfut.edu.cn, bhgdxjc@163.com
}

\begin{abstract}
Keywords: Social Media, New pattern of online shopping, Customer perceived value, Information quality, Brand preference
\end{abstract}

\begin{abstract}
In this paper, we remodel the dimensions of the customer perceived value. On the basis of the above, we demonstrate that the influence of customer perceived value on brand preference under the new pattern of online shopping, and put forward the social media information quality is regulating the relationship between customers perceived value and brand preference. Through theoretical analysis of the effect of social media on brand preference, this paper can provide theoretical support for managers on brand operation and marketing decisions in the new network ecological environment.
\end{abstract}

\section{社会化媒体对品牌偏好的影响机理： 基于顾客感知价值的视角}

\author{
丁 勇 $1, \mathrm{a}$, 肖金川1,b, \\ 1合肥工业大学管理学院, 合肥, 安徽, 中国 \\ adingyong@hfut.edu.cn, bhgdxjc@163.com
}

关键词: 社会化媒体; 网络购物新模式; 顾客感知价值; 信息质量; 品牌偏好

中文摘要：在重构顾客感知价值维度的基础上，论证了新网络购物模式下六维度顾客感知价 值对品牌偏好的影响机理，并提出社会化媒体信息质量正向调节顾客感知价值与品牌偏好的 影响关系。通过深入论证社会化媒体对品牌偏好的影响, 为企业经营者在新的网络生态环境 下进行品牌运营和营销决策提供一定的理论支撑。

\section{1.引言}

社会化媒体的发展与普及打破了传统的以 “电商平台”为主体的网络购物模式，衍生出 “社会化媒体+电商平台”购物新模式。它借助社交网络、微博、在线社区等传播途径辅助商 品的销售和购买, 极大地丰富了消费者的消费体验。相关研究表明, 消费者选择某品牌产品 时, 不仅会追求产品的真实属性与功能, 也会注重购买产品的过程是否使其身心舒畅, 是否 能满足其自我表现的需求, 或是否有助于其自身社会地位、形象的提升 ${ }^{[1,2]}$ 。当消费者对品牌 的感知逐渐升华到对品牌的认可和归属时便产生了品牌偏好。

品牌偏好的形成和维持会进一步引起消费者品牌选择上的倾斜, 是消费者形成品牌忠诚 的基础和前期阶段, 这不但有利于企业开拓市场, 传播正面形象, 而且有利于管理客户关系, 研究消费者的个性化需求，实施精准营销。然而，在产品同质化日趋严重的今天，消费者表 
现出来的消费行为和心理往往是 “三心二意” 的，他们倾向于在同一品类中尝试选择不同的 品牌, 而这一现象加剧了企业培养消费者偏好的难度。社会化媒体购物新时代的来临, 突显 了人与人的平等交互, 助推了个性化定制、柔性化生产的发展, 为培育消费者品牌偏好带来 新的挑战和机遇。

回顾相关研究，国内外研究学者多以一种社会化媒体 (如社交网站、虚拟社区或微博客) 为例, 探讨社会化媒体对消费者购买偏好、情感维系和品牌忠诚等方面的影响。黄京华等人 （2016）认为消费者感知的微博价值会促使其产生企业认同，进而影响其对企业和产品的关 系感知 ${ }^{[3]}$ 。Gamboa and Gonçalves (2014) 基于 Facebook 实证了感知价值与品牌忠诚度的关 系, 并认为 Facebook 的使用为培育消费者忠诚度提供了新的机会 ${ }^{[4]}$ 。金立印等学者的研究结 果显示顾客感知到的虚拟品牌社区价值可以提升消费者社群意识和消费满意度, 有利于品牌 与消费者之间的感情维系 ${ }^{[5-6]}$ 。然而, 单一的社会化媒体只能体现社会化媒体的部分功能特征。 例如, 微博的特征更多地表现在即时分享和广而告之上, 微信的特征则更多地体现在即时交 互和圈内传播上。随着时代的发展和社会化媒体的普及, 网络消费者接触的社会化媒体多种 多样, 对社会化媒体的感知也是综合性的, 以单一社会化媒体为例探讨社会化媒体对消费者 消费意向和品牌认知的影响存在一定的局限性。

本研究将社会化媒体看作统一的整体, 结合马斯洛需求层次理论和 Philip Kotler 的消费 者行为影响四层面论, 将顾客感知价值细分为社交价值、质量价值、服务价值、形象价值、 经济价值和利他价值六个维度, 并以社会化媒体信息质量作为顾客感知价值和品牌偏好关系 的调节变量, 探讨社会化媒体购物环境下六维顾客感知价值对品牌偏好的影响机理, 以期为 相关企业实施网络品牌营销提供借鉴和启示。

\section{2.相关概念及理论模型}

\section{1 社会化媒体购物与品牌偏好}

以顾客为中心的消费时代, 消费者对不同品牌的感知存在差异, 而品牌偏好正是在这种 差异化中形成的。相关研究认为, 品牌偏好是消费者对某一特定品牌产品或服务表现出来的 带有较强情感色彩和购买倾向的行为意向 ${ }^{[1,7]}$ 。在新媒体大爆炸的今天, 越来越多的网络用户 愿意借助社会化媒体渠道搜索、采集产品或服务的宣传信息和使用见解。相较传统网络购物 模式, 社会化媒体购物模式在交互性、参与性、透明性、引领性、集群性、连通性方面特征 突出。有研究学者对法国、英国和美国三个发达国家的消费者进行调查研究, 结果表明社会 化媒体的应用能正向影响顾客与品牌的关系质量 ${ }^{[8]}$ 。社会化媒体的应用提高了消费者与品牌 商家的互动性，积极影响消费者的购买意愿和品牌认可度 ${ }^{[9-10]}$ 。

\section{2 顾客感知价值与价值维度}

顾客感知价值是顾客购买产品或服务时权衡获得的利益与付出的成本后对产品或服务价 值的总体评价 ${ }^{[2,5]}$ 。其中, 顾客收获的利益包括可靠的产品、超值的服务和良好的品牌效应等, 而顾客付出的成本包括时间成本、精力成本和货币成本等。社会化媒体购物环境下，不同消 费个体的感知价值不仅会受到人口特征（如性别、年龄、受教育程度等）的影响，也会受到 消费个体对社会化媒体接受、依赖程度的影响 ${ }^{[13,24]}$ 。本研究结合社会化媒体购物新模式的特 征表现, 以马斯洛需求层次理论和 Philip Kotler 的消费者行为影响因素四层面说作为理论支 撑, 将顾客感知价值细分为社交价值、质量价值、服务价值、形象价值、经济价值和利他价 值六个维度。

\section{3 社会化媒体信息质量}

随着社会化媒体的发展，信息的交互更加频繁，信息的来源更加多源，信息的表现形式 也更加多样, 消费者只需要在互联网上进行简单的搜索点击即可获取海量信息资源。但是, 消费者的信息处理能力是有限的, 他们没有能力和精力对所有的信息进行分析处理, 然后做 
出决策。当消费者对品牌的信息进行检索时，通常会先对信息进行一个基本的判断和鉴定， 鉴定的结果将直接影响消费者对该品牌的感知态度。相关研究表明，信息的多元性、专业性、 即时性、透明性和新颖性越高意味着信息质量越高 ${ }^{[11,19,25]}$ 。信息质量越高越有利于品牌商家 和消费者建立伙伴关系，促进信任的发展和消费者忠诚度的提升 ${ }^{[11]}$ 。

\section{4 理论模型}

根据前人的研究结论以及本文的研究内容，为探讨社会化媒体对品牌偏好的影响，本文 提出了如图1所示的理论模型。其中，网络消费者的性别、年龄、学历和对社会化媒体持有的 接受、依赖态度作为影响顾客感知价值的宏观因素, 六维度顾客感知价值作为影响消费者品 牌偏好直接因素，社会化媒体信息质量作为顾客感知价值与品牌偏好关系的调节变量。

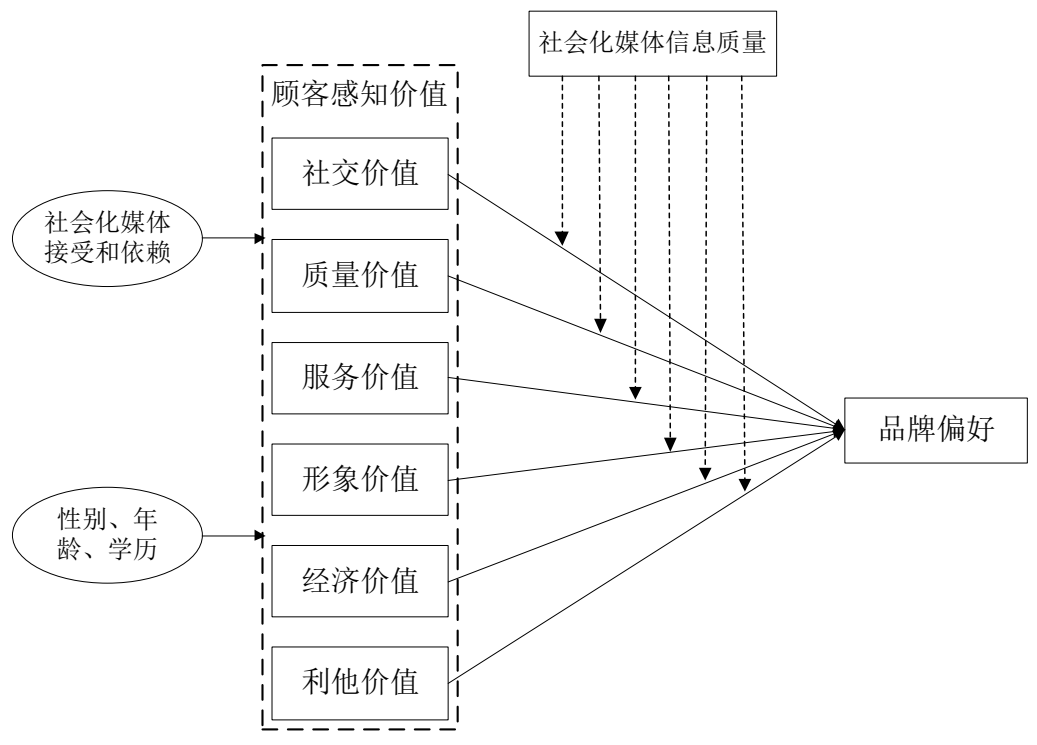

图 1 理论模型

\section{3.社会化媒体对品牌偏好的影响机理}

\section{1 顾客感知价值对品牌偏好的影响}

\section{1 .1 社交价值对品牌偏好的影响}

社会化媒体的社交功能满足了用户现实社会交往的需求，有助于人们在网络世界中找到 背景相同、品味相投的群体，实现网络社交由 “虚拟性” 向 “日常性” 的跨越。这种社会交 往活动的基础较为坚实，交流会更加频繁，彼此之间的信任也就越浓厚。由消费者组建的社 会关系网络有利于发挥信息的收集和传递功能，同时也是积累社会资本的过程 ${ }^{[12]}$ 。品牌社区 中的人际交互以及由此带来的信息和相关社会价值会影响一个人的购物决策及其购后行为 [13]。当消费者意识到通过参与某品牌的在线讨论或分享品牌信息能够满足自己的社交需求时, 消费者很大程度上会对该品牌产生较强的兴趣和情感依赖 ${ }^{[12]}$ 。

\section{1 .2 质量价值对品牌偏好的影响}

顾客感知质量价值是影响顾客制定购买决策和产生品牌偏好的重要因素 ${ }^{[7]}$ 。线索利用理 论认为, 消费者在面对材质、功能和性能均不确定的产品时, 消费者可能会没有能力或动力 进行系统性分析，而是利用启发式线索进行产品质量的判断。如果从不同信息源获取的产品 质量信息具有较强一致性时，消费者会因此产生较强烈的品牌信任和认可度。社会化媒体购 物环境下, 消费者虽不能直接接触产品, 但可以依赖更多的外部线索（例如质量保证、商家 名誉和使用者的使用体验等）对产品质量进行感知。感知质量的好坏影响消费者心中的品牌 印象, 品牌印象进一步反映消费者的品牌态度。

3.1.3 服务价值对品牌偏好的影响

随着收入水平的提高和消费观念的改变，消费者在选购产品时，不仅会注意产品本身价 
值的高低，也会考虑产品附加价值（如服务）的大小。投放到市场上的产品如果缺乏服务的 保证, 将难以吸引持续不断的消费者。优质的顾客服务会提升消费者消费满意度, 并能增强 消费者再次光顾的意愿。有用的、及时的在线响应能力对网络购物满意度有积极影响, 并能 提升消费者忠诚度 ${ }^{[14]}$ 。在社会化媒体购物环境下，消费者对服务的精准性、个性化、即时性 要求越来越高, 随着市场竞争的加剧, 在线服务质量的高低将直接影响消费者的购买决策和 品牌好感度。

3.1.4 形象价值对品牌偏好的影响

消费者将是否能够更好地呈现自我价值观和自我塑造的社会形象，作为制定购买决策、 产生品牌好感的衡量指标之一 ${ }^{[15]}$ 。消费者自我概念与品牌形象越一致, 消费者对品牌的态度 也就越积极, 关注和购买这一品牌的意愿也就越强烈。在社会化媒体购物环境下, 消费者在 汶览品牌信息或参与品牌互动时, 会潜意识地将品牌形象与自我形象进行比照。如果消费者 能够感知到该品牌提供的产品或服务与自身的社会地位和自我形象相符或能提升自身的社会 地位和自我形象, 则出于对自身心理需求的满足, 消费者就会对该品牌产生一定的情感依赖 [22]。

\subsection{5 经济价值对品牌偏好的影响}

在现实生活中，顾客通过付出一定的货币、时间和精力成本来获取产品或服务。对于一 些价格敏感度高的消费者而言, 货币方面的付出是影响消费偏好形成的关键性因素; 对于那些 价格敏感度低的消费者而言, 减少时间、精力方面的支出则显得更为重要 ${ }^{[1]}$ 。社会化媒体购物 环境下，品牌商家提供的即时促销活动、优惠券和多渠道的销售模式等能直接节省消费者的 货币、时间或精力方面的支出。在产品同质化日趋严重的今天，相似产品或服务多种多样， 经济上的优惠正在成为在线消费者选择产品, 产生重复购买行为, 形成品牌偏好的重要因素 之一[10]。

\subsection{6 利他价值对品牌偏好的影响}

利他价值是指顾客感知自身的努力或参与为他人、企业、社会或环境带来积极影响的效 用。相关研究揭示, 消费者在购买产品时, 除了考虑个人收益外, 利他主义因素也在左右消 费者的购买偏好 ${ }^{[17]}$ 。当消费者想要向他人或社会展现正义的、积极的自我形象时, 消费者会 对利他诉求表现出更为正面的意向和行为 ${ }^{[17]}$ 。品牌商家对环境、社会和利益相关者的责任担 当积极影响消费者品牌偏好的形成 ${ }^{[7]}$ 。在社会生活比较繁荣稳定时, 人们乐于追求优越感、 争取成功, 希望通过自身的参与为社会做出更多的贡献。在社会化媒体购物环境下, 得当的 品牌社会形象和社会担当宣传更易激发消费者利他主义行为, 引起消费者集群共鸣的同时也 会加深消费者的品牌印象 ${ }^{[18]}$ 。另外，品牌商家对消费者参与、分享行为的认可和激励也会进 一步激发消费者利他主义行为, 促使更多的消费者愿意携手品牌商家共创美好未来 ${ }^{[10,16] 。}$

\section{2 信息质量的调节作用}

网络购物环境下，信息多样性、丰富性、可获得性的增强会对消费者购买决策和品牌信 任产生显著影响 ${ }^{[19]}$ 。消费者根据所处的网络购物环境和自身认知能力, 选择性的对品牌信息 进行处理, 进而形成一定的品牌印象。在这一过程中高质量的信息很容易吸引消费者的注意, 从而影响消费者的购买倾向和品牌好感度。社会化媒体购物模式下, 消费者的消费行为和品 牌态度贯穿于信息搜索、信息浏览、信息获取、信息利用和信息自生成的始终。高质量的信 息能提升在线消费者的持续参与度、服务满意度和质量信任感，有利于消费者即时捕捉有用 信息, 强化消费者自我概念与品牌形象的一致性, 激发消费者利他主义需求, 进而影响在线 消费者参与品牌建设、抵制竞争品牌的积极意向 ${ }^{[20-21,23]}$ 。因此社会化媒体信息通过多元性、 专业性、即时性、透明性和新颖性等特征从细节上影响顾客感知价值和品牌偏好的关系, 进 而影响消费者的购买意向和消费行为。 


\section{4.营销管理启示}

社会化媒体的发展和普及给企业品牌建设带来前所未有的挑战和机遇。企业要想在互联 网技术快速发展的今天生存并取得持续的效益, 基于新媒介、新手段的品牌建设已经成为一 个不可回避的话题。所以本研究相关内容对于企业进行品牌营销策划, 提升品牌影响力具有 一定的启示意义。

（1）充分利用社会化媒体资源，精准挖掘顾客需求价值，构建科学完善的顾客感知价值 体系, 并维持顾客感知价值体系的时效性和动态变化性。结合现阶段顾客感知价值体系, 企 业可以借助社会化媒体优势, 合理分配营销资源和营销渠道, 专注推广质量稳定、性能优越 的产品, 并以鲜明的品牌形象和专题研讨加以烘托和辅助, 使产品质量和品牌形象深入人心; 同时, 企业应在力所能及的范围内努力提升社会责任水平, 寻求多方合作, 拓展社会责任活 动范围, 进一步加深消费者对企业品牌的认可程度，提升品牌好感度。另外，从价值层面来 讲品牌影响力的培养是发现价值、塑造价值和传递价值的过程。随着社会的进步和互联网技 术的发展, 人们的消费观念也在不断变化, 只有保持顾客感知价值体系的时效性和动态变化 性才能适时迎合、甚至引领消费者的消费需求。

（2）结合社会化媒体的功能特征，不断完善丰富信息质量指标体系，并进一步加强在线 服务人员的专业技能和礼仪规范的培训。随着社会化媒体的快速发展，信息碎片化现象日益 严重, 海量的信息和快节奏地生活迫使消费者只能有选择地接受和吸收少部分的信息。企业 需要深度挖掘消费者的核心需求，并结合相应的信息质量指标体系对品牌信息进行加工再创 造, 创造出价值凸显、新意盎然、通俗易懂又不失规范的信息来吸引消费者的注意。另外, 社会化媒体的应用和普及使得消费者与企业的沟通更加扁平化，在线服务人员应主动借助社 会化媒体寻找有关企业品牌产品的讨论或者埋怨，对消费者提出的问题或建议做出回应，对 产品的质疑做出专业性的答复, 有针对性地解决问题, 重视顾客消费体验的完整性, 聆听他 们的意见并做出反馈, 给顾客留下负责任的企业形象, 以加深消费者对企业品牌的印象和好 感度。

\section{致谢}

本文为教育部人文社会科学规划基金项目《社会化媒体对企业绩效的影响机制研究》 (15YJA630010)的阶段性成果之一。

\section{References}

[1] J W Overby, E J Lee. The effects of utilitarian and hedonic online shopping value on consumer preference and intentions, Int. J. Journal of Business research, vol. 59, pp. 1160-1166, 2006.

[2] J C Sweeney, G N Soutar. Consumer perceived value: The development of a multiple item scale, Int. J. Journal of retailing, vol. 77, pp. 203-220, 2001.

[3] J Huang, Y Jin, J Zhang. How Can Enterprise Microblogging Improve Consumer Loyalty? An Empirical Study Based on Social Identity Theory, Int. J. Nankai Business Review, vol. 19, pp. 159-168, 2016.

[4] A M Gamboa, H M Gonçalves. Customer loyalty through social networks: Lessons from Zara on Facebook, Int. J. Business Horizons, vol. 57, pp. 709-717, 2014.

[5] L Jin. The Effects of Value Dimensions on Sense of Community, Loyalty and Brand Related Behavior Intentions in Virtual Brand Community, Int. J. Journal of Management Science, vol. 20, pp. 36-45, 2007.

[6] M Royo-Vela, P Casamassima. The influence of belonging to virtual brand communities on consumers' affective commitment, satisfaction and word-of- mouth advertising: The ZARA 
case, Int. J. Online Information Review, vol. 35, pp. 517-542, 2011.

[7] R Chu, G Shi, J L Brock, et al. The impact of corporate social responsibility (CSR) performance and perceived brand quality on customer-based brand preference, Int. J. Journal of Services Marketing, vol. 28, pp. 181-194, 2014.

[8] S Hudson, L Huang, M S Roth, et al. The influence of social media interactions on consumerbrand relationships: A three-country study of brand perceptions and marketing behaviors, Int. J. International Journal of Research in Marketing, vol. 33, pp. 27-41, 2015.

[9] B Bickart, R M Schindler. Internet forums as influential sources of consumer information, Int. J. Journal of interactive marketing, vol. 15, pp. 31-40, 2001.

[10] J Dong, J Chen. Xiaomi Business Model Innovation: redefining the relationship Between a Manufacturer and Its Customers, Int. J. China Soft Science, vol. , pp. 22-33, 2015.

[11] Y Salaün, K Flores. Information quality:: Meeting the needs of the consumer, Int. J. International Journal of Information Management, vol. 21, pp. 21-37, 2001.

[12] X Wang, H Xue. Brand Community Social Capital, Perceived Value and Brand Loyalty, Int. J. Journal of Management Science, vol. 23, pp. 53-63, 2011.

[13] H Chen. The impact analysis of social network structure on the consumer behavior, Int. J. Statistics and Decision, vol., pp. 94-97, 2016.

[14] J Kim, B Jin, J L Swinney. The role of e-tail quality, e-satisfaction and e-trust in online loyalty development process, Int. J. Journal of Retailing \& Consumer Services, vol. 16, pp. 239-247, 2009.

[15] F Liu, J Li, D Mizerski, et al. Self-congruity, brand attitude, and brand loyalty: a study on luxury brands, Int. J. European Journal of Marketing, vol. 46, pp. 922-937, 2012.

[16] Y Peng. A Theoretical Frame of Management of Customer Participation during Value Co-creation with Enterprises, Int. J. China Business and Market, vol. 28, pp. 70-76, 2014.

[17] K White, J Peloza. Self-benefit versus other- benefit marketing appeals: Their effectiveness in generating charitable support, Int. J. Journal of Marketing, vol. 73, pp. 109-124, 2009.

[18] Z Cao, Q Dai, X Zhao. The Effects of Company-consumer Fit and Communication Focus in Cause-related Marketing, Int. J. Nankai Business Review, vol. 15, pp. 62-71, 2012.

[19] J R Coyle, E Thorson. The Effects of Progressive Levels of Interactivity and Vividness in Web Marketing Sites, Int. J. Journal of Advertising, vol. 30, pp. 65-77, 2001.

[20] G Li, Y Li, F Ni. Online Community E-Commerce: An Empirical Study Based on Members' Intention to Online transaction, Int. J. Management Review, vol. 23, pp. 78-86, 2011.

[21] C Qiu, K Sun, A Gu. Research on Theoretical Model of Effect of Brand Information Content Display on Consumer Participation in the Mobile Internet Context, Int. J. Library and Information Service, vol. 60, pp. 40-46, 2016.

[22] J Lu, Y Xu. Chinese young consumers' brand loyalty toward sportswear products: a perspective of self-congruity, Int. J. Journal of Product \& Brand Management, vol. 24, pp. 365-376, 2015.

[23] N Bi, C Yin, H Lan. Experimental Research on Customer Response to Information Value_-Using Wechat as an Example, Int. J. Information Science, vol. , pp. 93-97, 2015.

[24] W Jiao, Z Wang. Online shopping using social media Impact factor: theoretical model building, Int. J. Research On Financial And Economic Issues, vol. , pp. 119-125, 2014. 
[25] W Zhao, R Chen. Research on credibility evaluation model of online information in social media, Int. J. Information studies: Theory \& Application, vol. 38, pp. 68-72, 2015. 